\title{
Prognostic value of preoperative hyponatremia in patients with epithelial ovarian cancer
}

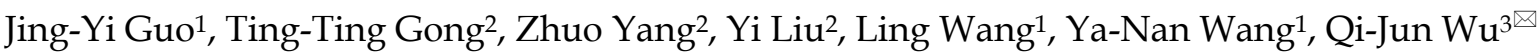 \\ 1. Department of postgraduate, Shengjing Hospital of China Medical University, Shenyang, China (J-YG, LW, and Y-NW). \\ 2. Department of Obstetrics and Gynecology, Shengjing Hospital of China Medical University, Shenyang, China (T-TG, ZY, and YL). \\ 3. Department of Clinical Epidemiology, Shengjing Hospital of China Medical University, Shenyang, China (Q-JW). \\ $\triangle$ Corresponding author: Qi-Jun Wu, M.D., Ph.D., Department of Clinical Epidemiology, Shenging Hospital of China Medical University. Address: No.36, San \\ Hao Street, Shenyang, Liaoning 110004, P. R. China. Phone: +86-24-96615-13648; Fax: +86-24-96615-13648; E-mail address: wuqj@sj-hospital.org \\ (C) Ivyspring International Publisher. This is an open access article distributed under the terms of the Creative Commons Attribution (CC BY-NC) license \\ (https://creativecommons.org/licenses/by-nc/4.0/). See http://ivyspring.com/terms for full terms and conditions.
}

Received: 2018.06.26; Accepted: 2018.10.21; Published: 2019.01.29

\begin{abstract}
Hyponatremia is an emerging issue in cancer patients. However, few studies have explored the prognostic value of preoperative hyponatremia in epithelial ovarian cancer (EOC) patients. We conducted a retrospective study of women diagnosed with EOC at Shengjing Hospital from 2011 to 2015. Preoperative serum sodium levels were measured and classified as normal $(136-145 \mathrm{mmol} / \mathrm{L})$ or hyponatremic $(\leq 135 \mathrm{mmol} / \mathrm{L})$ according to the average/most recent measurements of this biomarker. Multivariable Cox proportional hazard regression models were applied to determine the adjusted hazard ratios (HRs) with $95 \%$ confidence intervals (Cls) for the association of preoperative hyponatremia with progression-free survival (PFS) and overall survival (OS). We identified $607 \mathrm{EOC}$ patients meeting the inclusion criteria with a median age of 51 years (inter-quartile range: 47-59 years). The median follow-up duration was 3.1 years (inter-quartile range: $2.2-4.2$ ). The recurrence and mortality rates were $56.8 \%(345 / 607)$ and $38.4 \%$ (233/607), respectively. Among the patients, 81 (13.3\%) were observed as hyponatremic. Preoperative hyponatremia was associated with poorer PFS $(\mathrm{HR}=1.51$; $95 \% \mathrm{Cl}=1.07-2.15)$ as well as $\mathrm{OS}(\mathrm{HR}=1.47 ; 95 \% \mathrm{Cl}=1.03-2.11)$ after adjusting for potential confounders. Notably, results were in line with the main findings only when using the most recent serum sodium levels before treatment, with corresponding HRs of $1.58(95 \% \mathrm{Cl}=1.12-2.23)$ for PFS and $1.45(95 \% \mathrm{Cl}=1.02-2.07)$ for OS. Preoperative hyponatremia is an independent prognostic factor of EOC. Further studies are warranted to confirm our findings as well as to determine whether correction of preoperative hyponatremia may alter clinical outcomes in these patients.
\end{abstract}

\section{Introduction}

Epithelial ovarian cancer (EOC) is the fourth leading cause of female cancer death in the developed world [1]. More than $80 \%$ of EOC patients have the advanced stage of the disease at the first diagnosis, with 5-year survival rates between $15 \%-45 \%$, which are extremely low among all types of cancers [1]. As a result of the poor prognosis associated with this disease, a number of laboratory tests including CA-125 [2], serum albumin [3,4], and C-reactive protein [5] have been analyzed in an attempt to find an effective diagnostic biomarker.

Hyponatremia is the most common electrolyte abnormality among hospitalized patients, with frequencies varying from $15 \%-20 \%$ for hospitalized patients [6] and $47 \%$ for cancer patients [7]. Although not directly linked with death, hyponatremia has been identified to be associated with increased morbidity and mortality [8]. Moreover, it was observed that aggressive management of hyponatremia aided anti-cancer treatment and improves survival [9]. Across different types of cancer, the impact of hyponatremia has been most frequently studied for small cell lung cancer due to its well-known underlying cause, namely syndrome of inappropriate antidiuretic hormone (SIADH). In recent years, potential influence of hyponatremia on disease prognosis has also been studied for some other common cancers, such as breast and colorectal cancer [10]. To our knowledge, few studies have been performed to examine the prevalence and prognostic 
value of hyponatremia in EOC. The most recent study performed by Martin and colleagues [11] investigated the relationship between preoperative hyponatremia and the prognosis of women with ovarian, fallopian tube, and primary peritoneal cancers using the American College of Surgeons National Surgical Quality Improvement Program database, and observed a prevalence of $10.02 \%(365 / 3644)$ and a positive association between preoperative hyponatremia and lengthening of hospital stays, 30-day postoperative mortality rates, and postoperative complications [11]. Nevertheless, whether preoperative hyponatremia may serve as a prognostic marker for EOC progression and long-term survival remains elusive.

To further clarify this issue, we undertook a retrospective cohort study to examine the association between preoperative hyponatremia and progression-free survival (PFS) and overall survival (OS) among EOC patients, using data from a single center hospital.

\section{Materials and Methods}

\section{Study design and patients}

The present retrospective cohort study enrolled EOC patients admitted consecutively to Shengjing Hospital of China Medical University between December 1, 2011 and December 31, 2015. The study was approved by the Institutional Review Board of the Ethics Committee of Shengjing Hospital (2015PS38K).

Patients were included if they: 1) were over 15 years of age; 2 ) had been diagnosed with primary EOC; 3 ) had undergone a surgical procedure; and 4) had a serum sodium measurement prior to surgery. In contrast, patients were excluded if they: 1) had undergone surgical exploration at other institutions but had received chemotherapy at Shengjing Hospital; 2) had received neoadjuvant therapy/unknown treatment; 3) had not received primary surgery; 4 ) had a preoperative serum sodium measurement that indicated hypernatremia; 5) had a missing preoperative serum sodium measurement within the 90 days prior to surgery; or 6) had missing information for any of the variables or covariates used in the analyses.

\section{Data collection}

Information on demographic and clinical factors was extracted manually through medical records from the hospital information system of Shengjing Hospital. Clinico-pathological data included the date of diagnosis, tumor histology, tumor grade, International Federation of Gynecology and Obstetrics (FIGO) stage, comorbidity, residual disease, and ascites. Tumor stage was calculated on the basis of the FIGO criteria [12]. Tumors were graded as well (G1), moderately (G2), or poorly (G3) differentiated according to the histological typing system of the WHO [13]. In addition, residual disease was grouped into "non-detectable", " $\leq 1 \mathrm{~cm}$," or " $>1$ cm." Performance status (PS) was assessed in accordance with the criteria of the Eastern Cooperative Oncology Group's (ECOG) scale [14]. Comorbidity, defined as the presence of one or more diseases in addition to the primary disease, was classified as "yes" or "no" according to the Charlson comorbidity index [15]. Histology was classified as 'serous' and 'non-serous'. All of the aforementioned data were collected and confirmed by two experienced gynecologists.

Of particular interest in this study was the average serum sodium levels provided by the hospital's laboratory center and measured within 90 days prior to surgery. Serum sodium was measured by the electrode method on an Abbott c16200 automatic biochemistry analyzer. We categorized serum sodium levels as normal (136-145 mmol/L) or hyponatremic $(\leq 136 \mathrm{mmol} / \mathrm{L})$.

\section{Outcomes}

To evaluate the clinical progression of disease, we manually extracted the data for clinical examinations, serum CA-125 assays, chest x-rays, abdominal-pelvic ultrasounds, and computed tomography scans from the medical records under the criteria of Response Evaluation Criteria in Solid Tumors. The primary endpoint was PFS, defined as the time from the completion of primary surgery to the first progression or recurrence of disease or death from any cause. OS was defined as the time from the completion of primary surgery to death from any cause or the date of last follow-up (December 31, 2017) for patients who were alive. The cause of death was obtained from the death certificates.

\section{Statistical analysis}

The relationship between preoperative hyponatremia and the demographic and clinico-pathological characteristics of EOC was analyzed using the Mann-Whitney $U$ test for continuous variables and the chi-square test for categorical variables. Continuous variables were summarized as the median with the inter-quartile range (IQR). Categorical variables were expressed as the number with the percentage. The Cox proportional hazards model was applied to calculate hazard ratios (HRs) and 95\% confidence intervals (CIs) for the association between preoperative hyponatremia and PFS and OS. We assessed the 
proportional hazards assumption with a likelihood ratio test.

We conducted multivariable adjusted analyses, including the following potential confounders: age at diagnosis, FIGO stage, histology, residual disease, comorbidity, performance status, ascites, cancer grading, and preoperative serum potassium level. Furthermore, we carried out subgroup analyses stratified by FIGO stage, residual disease, and comorbidity. Likelihood ratio tests were conducted to examine whether the associations between preoperative hyponatremia and PFS and OS were modified by these aforementioned prespecified potential effect modifiers. In sensitivity analyses, we treated the exposure of interest as the most recent serum sodium measured in the 90 days prior to surgery. We also excluded patients who showed recurrence of disease or died within 1 year of study enrollment. All analyses were performed using SAS software, version 9.3 (SAS Institute, Cary, NC, USA).

\section{Results}

In total, 1390 women with ovarian, primary peritoneal cancers, and fallopian tube cancers were identified at Shengjing Hospital from December 1, 2011 to December 31, 2015. Of these patients, the following were excluded from the study: 47 women who were diagnosed with primary peritoneal cancers and fallopian tube cancers, 159 patients who had received neoadjuvant chemotherapy, 221 patients who had not undergone primary surgery/underwent surgical exploration at another institution, 14 patients aged younger than 15, 114 patients who had hypernatremia, and 228 patients who had missing information for any of the variables or covariates. Finally, the remaining 607 women with primary EOC and a median age of 51 (IQR: 47-59) were included in the study.

The selected demographic and clinical characteristics of EOC patients according to preoperative serum sodium levels are listed in Table 1. The median follow-up duration was 3.1 years (IQR: 2.2-4.2), with $345(56.8 \%)$ patients showing disease recurrence and $233(38.4 \%)$ patients dying during this period. The median PFS and OS of all patients were 2.5 years (IQR: 1.2-3.9 years) and 3.1 years (IQR: 2.2-4.2 years), respectively. Over half of the patients were diagnosed without a comorbidity (336 patients, $55.4 \%$ ). The majority of these cases (458 patients, $75.5 \%$ ) displayed a bad performance status (PS $\geq 2$ ). A total of $354(58.3 \%)$ patients with EOC were categorized as FIGO stage III/IV. However, interestingly, over one quarter $(n=161)$ of EOC patients were categorized as FIGO stage I. The pathology of the 607 patients consisted of 434 (71.5\%) serious EOC cases and 173 (28.5\%) non-serious EOC cases. After surgery, $80.2 \%(487 / 607)$ of cases had a residual tumor size of less than $1 \mathrm{~cm}$. In addition, high grade tumors $(n=413,68 \%)$ accounted for the majority of tumors in these EOC patients. A total of 81 patients $(13.3 \%)$ were diagnosed with hyponatremia in this study. However, we failed to detect any significant differences between normal and hyponatremia patients in relation to demographic and clinical characteristics. Table 2 summarizes selected patient characteristics in relation to PFS and OS after mutual adjustment. Possessing a comorbidity, residual disease, and advanced FIGO stage (III/IV) was associated with poorer PFS and OS of EOC patients.

Table 1. Selected demographic and clinical characteristics of epithelial ovarian cancer patients according to the average preoperative serum sodium levels measured in the 90 days prior to surgery

\begin{tabular}{|c|c|c|c|c|}
\hline Variables & Overall & Normal & Hyponatremia & $\begin{array}{l}P \\
\text { value } \dagger\end{array}$ \\
\hline No. of patients & 607 & 526 & 81 & \\
\hline $\begin{array}{l}\text { Age at diagnosis (years), } \\
\text { Median (IQR) }\end{array}$ & $51(47-59)$ & $51(47-59)$ & $52(47-59)$ & 0.44 \\
\hline $\begin{array}{l}\text { Follow-up time (years), } \\
\text { Median (IQR) }\end{array}$ & $\begin{array}{l}3.1 \\
(2.18-4.21)\end{array}$ & $\begin{array}{l}3.02 \\
(2.18-4.26)\end{array}$ & $2.91(2.16-3.80)$ & 0.27 \\
\hline $\begin{array}{l}\text { Serum potassium (mmol/L), } \\
\text { Median (IQR) }\end{array}$ & $\begin{array}{l}2.54 \\
(1.21-3.88)\end{array}$ & $\begin{array}{l}4.06 \\
(3.84-4.29)\end{array}$ & $4.29(3.86-4.59)$ & 0.15 \\
\hline $\begin{array}{l}\text { Serum albumin }(g / L) \text {, } \\
\text { Median (IQR) }\end{array}$ & $\begin{array}{l}41 \\
(36.7-44.1)\end{array}$ & $\begin{array}{l}41 \\
(36.7-44.1)\end{array}$ & $41(37-43.4)$ & 0.83 \\
\hline $\begin{array}{l}\text { Serum creatinine }(\mu \mathrm{mol} / \mathrm{L}) \text {, } \\
\text { Median (IQR) }\end{array}$ & $\begin{array}{l}54.1 \\
(48.5-60.9)\end{array}$ & $\begin{array}{l}54 \\
(48.5-61.1)\end{array}$ & $55.3(48.1-60.2)$ & 0.93 \\
\hline Vital status (\%) & & & & 0.07 \\
\hline Alive & $374(61.6)$ & $332(63.1)$ & $42(51.8)$ & \\
\hline Died & $233(38.4)$ & $194(36.9)$ & $39(48.2)$ & \\
\hline Recurrence status (\%) & & & & 0.09 \\
\hline Yes & $345(56.8)$ & $306(58.2)$ & $39(48.2)$ & \\
\hline No & $262(43.2)$ & $220(41.8)$ & $42(51.8)$ & \\
\hline Comorbidity $(\%)$ & & & & 0.78 \\
\hline No & $336(55.4)$ & $290(55.1)$ & $46(56.8)$ & \\
\hline Yes & $271(44.6)$ & $236(44.9)$ & 35 (43.2) & \\
\hline Performance status ( $\%)$ & & & & 0.57 \\
\hline 0 & $4(0.7)$ & $4(0.8)$ & 0 & \\
\hline 1 & $145(23.9)$ & $125(23.7)$ & $20(24.7)$ & \\
\hline 2 & $302(49.8)$ & $265(50.4)$ & $37(45.7)$ & \\
\hline$\geq 3$ & $156(25.6)$ & $132(25.1)$ & $24(29.6)$ & \\
\hline FIGO stage $(\%)$ & & & & 0.59 \\
\hline I & $161(26.5)$ & $144(27.4)$ & $17(21)$ & \\
\hline II & $92(15.2)$ & $78(14.8)$ & $14(17.3)$ & \\
\hline III & $313(51.6)$ & $270(51.3)$ & $43(53.1)$ & \\
\hline IV & $41(6.7)$ & $34(6.5)$ & $7(8.6)$ & \\
\hline Histologic type (\%) & & & & 0.06 \\
\hline Serous & $434(71.5)$ & $369(70.2)$ & $65(80.3)$ & \\
\hline Non-serous & $173(28.5)$ & $157(29.8)$ & $16(19.7)$ & \\
\hline Residual disease (\%) & & & & 0.81 \\
\hline None detectable & $373(61.4)$ & $321(61)$ & $52(64.2)$ & \\
\hline$\leq 1 \mathrm{~cm}$ & $114(18.8)$ & $99(18.8)$ & $15(18.5)$ & \\
\hline$>1 \mathrm{~cm}$ & $120(19.8)$ & $106(20.2)$ & 14 (17.3) & \\
\hline Ascites (\%) & & & & 0.26 \\
\hline Yes & $257(42.3)$ & $218(41.4)$ & $39(48.2)$ & \\
\hline No & $350(57.7)$ & $308(58.6)$ & $42(51.8)$ & \\
\hline Grading (\%) & & & & 0.26 \\
\hline Grade 1 & $31(5.1)$ & $25(4.8)$ & $6(7.4)$ & \\
\hline Grade 2 & $163(26.9)$ & $137(26.1)$ & $26(32.1)$ & \\
\hline Grade 3 & $413(68)$ & $364(69.1)$ & $49(60.5)$ & \\
\hline
\end{tabular}


Table 2. Selected demographic and clinical characteristics according to progression-free survival and overall survival among epithelial ovarian cancer patients

\begin{tabular}{|c|c|c|c|c|}
\hline \multirow[t]{2}{*}{ Variables } & \multicolumn{2}{|l|}{ PFS } & \multicolumn{2}{|l|}{ OS } \\
\hline & No./Events & HR $(95 \% \mathrm{CI})^{\dagger}$ & No./Events & HR $(95 \% \mathrm{CI}) \dagger$ \\
\hline \multicolumn{5}{|l|}{ Age at diagnosis } \\
\hline$\leq 50$ & $266 / 110$ & 1.00 (Ref) & $266 / 96$ & 1.00 (Ref) \\
\hline$>50$ & $341 / 152$ & $1.24(0.96-1.60)$ & $341 / 137$ & $1.27(0.97-1.66)$ \\
\hline \multicolumn{5}{|l|}{ Comorbidity } \\
\hline No & $336 / 132$ & 1.00 (Ref) & $336 / 111$ & 1.00 (Ref) \\
\hline Yes & $271 / 130$ & $1.42(1.10-1.82)$ & $271 / 122$ & $1.58(1.22-2.59)$ \\
\hline \multicolumn{5}{|c|}{ Performance status } \\
\hline $0-1$ & $153 / 62$ & 1.00 (Ref) & $153 / 52$ & 1.00 (Ref) \\
\hline 2 & $302 / 118$ & $1.00(0.72-1.38)$ & $302 / 104$ & $1.19(0.84-1.68)$ \\
\hline$\geq 3$ & $152 / 82$ & $1.27(0.88-1.83)$ & $152 / 77$ & $1.35(0.92-1.98)$ \\
\hline \multicolumn{5}{|l|}{ FIGO stage (\%) } \\
\hline I-II & $253 / 52$ & 1.00 (Ref) & $253 / 45$ & 1.00 (Ref) \\
\hline III & $313 / 176$ & $2.50(1.75-3.58)$ & $313 / 153$ & $2.35(1.62-3.42)$ \\
\hline IV & $41 / 34$ & $7.05(4.42-11.24)$ & $41 / 35$ & $7.77(4.80-12.57)$ \\
\hline \multicolumn{5}{|c|}{ Histologic type $(\%)$} \\
\hline Serous & $434 / 214$ & 1.00 (Ref) & $434 / 185$ & 1.00 (Ref) \\
\hline Non-serous & $173 / 48$ & $0.79(0.57-1.11)$ & $173 / 48$ & $0.98(0.70-1.38)$ \\
\hline \multicolumn{5}{|l|}{ Residual disease } \\
\hline None detectable & $373 / 113$ & 1.00 (Ref) & $373 / 91$ & 1.00 (Ref) \\
\hline$\leq 1 \mathrm{~cm}$ & $114 / 68$ & $1.72(1.24-2.39)$ & $114 / 64$ & $2.19(1.56-3.09)$ \\
\hline$>1 \mathrm{~cm}$ & $120 / 81$ & $1.96(1.42-2.69)$ & $120 / 78$ & $2.50(1.79-3.47)$ \\
\hline \multicolumn{5}{|l|}{ Ascites } \\
\hline No & $257 / 132$ & 1.00 (Ref) & $257 / 123$ & 1.00 (Ref) \\
\hline Yes & $350 / 130$ & $1.06(0.81-1.39)$ & $350 / 110$ & $1.24(0.93-1.65)$ \\
\hline \multicolumn{5}{|l|}{ Grading } \\
\hline Grade 1 & $31 / 10$ & 1.00 (Ref) & $31 / 8$ & 1.00 (Ref) \\
\hline Grade 2 & $163 / 59$ & $1.11(0.57-2.17)$ & $163 / 50$ & $1.73(0.82-3.64)$ \\
\hline Grade 3 & $413 / 193$ & $1.42(0.70-2.87)$ & $413 / 175$ & $2.02(0.93-4.39)$ \\
\hline
\end{tabular}

$\mathrm{CI}$, confidence interval; FIGO, International Federation of Gynecology and Obstetrics; HR, hazard ratio; OS, overall survival; PFS, progression-free survival. tHRs (95\% CIs) for progression-free survival and overall survival were estimated by using multivariable proportional hazard models, mutually adjusted for all other variables listed in the table.

Overall, preoperative hyponatremia showed a $51 \%$ reduction in PFS of EOC patients compared with normal patients ( $\mathrm{HR}=1.51,95 \% \mathrm{CI}=1.07-2.15)$ after adjustment for potential confounders (Table 3). Similarly, there was a $47 \%$ reduction in OS of EOC patients compared with normal patients $(\mathrm{HR}=1.47$, 95\% CI=1.03-2.11) in the multivariable model. Of note, in the subgroup analyses, associations were slightly more marked in FIGO IV patients (for PFS: $\mathrm{HR}=5.89,95 \% \mathrm{CI}=1.31-26.54$; for $\mathrm{OS}: \mathrm{HR}=3.76$, 95\% $\mathrm{CI}=1.04-13.54)$, in EOC patients with non-detectable residual disease (for PFS: $\mathrm{HR}=1.74,95 \% \mathrm{CI}=1.05-2.88$ ), and in patients with $>1 \mathrm{~cm}$ residual disease (for PFS: $\mathrm{HR}=2.17,95 \% \mathrm{CI}=1.04-4.51$ ). There was no significant interaction effect detected for these variables ( $\mathrm{P}$ for interaction $>0.05$ ).

In sensitivity analyses, we treated the exposure of interest as the most recent serum sodium measurement taken within the 90 days prior to surgery (Table 4). These results were similar to the main findings. Furthermore, when we excluded 30 patients who showed recurrent disease within 1 year of study enrollment and 46 patients who died within 1 year of study enrollment, the results were still robust (data not shown).

\section{Discussion}

In this retrospective cohort study of 607 EOC patients, we observed a $51 \%$ and $47 \%$ reduction in the PFS and OS of EOC patients who had preoperative hyponatremia, respectively. These findings were robust in the subgroup and sensitivity analyses.

Hyponatremia has been identified as a negative prognostic factor in several malignancies $[10,16]$. However, recent studies on the prognosis of hyponatremia in EOC are limited. In 2015, Abu and coworkers [16] evaluated the potential prognostic value of hyponatremia in EOC by reviewing the medical records of 2,048 patients with more than 20 types of cancers (48 with ovarian cancer) admitted to the National Center for Cancer Care and Research in Qatar between 2008 and 2012.

Table 3. Hazard ratio $(95 \% \mathrm{Cl})$ for progression-free survival and overall survival among epithelial ovarian cancer patients according to the average preoperative serum sodium levels measured in the 90 days prior to surgery

\begin{tabular}{|c|c|c|c|c|c|c|}
\hline & Progression-fr & urvival & & Overall surviv & & \\
\hline & Normal & Hyponatremia & $P$ value for & Normal & Hyponatremia & $P$ value for \\
\hline & HR $(95 \% \mathrm{CI}) \dagger$ & HR $(95 \% \mathrm{CI}) \dagger$ & Interaction & HR $(95 \% \mathrm{CI}) \dagger$ & HR $(95 \% \mathrm{CI}) \dagger$ & Interaction \\
\hline All patients & 1.00 (Ref) & $1.51(1.07-2.15)$ & & 1.00 (Ref) & $1.47(1.03-2.11)$ & \\
\hline FIGO stage & & & 0.07 & & & 0.06 \\
\hline I & 1.00 (Ref) & $1.33(0.27-6.56)$ & & 1.00 (Ref) & $1.98(0.39-10.02)$ & \\
\hline II & 1.00 (Ref) & $1.65(0.67-4.07)$ & & 1.00 (Ref) & $2.15(0.81-5.74)$ & \\
\hline III & 1.00 (Ref) & $1.44(0.92-2.25)$ & & 1.00 (Ref) & $1.33(0.83-2.13)$ & \\
\hline IV & 1.00 (Ref) & $5.89(1.31-26.54)$ & & 1.00 (Ref) & $3.76(1.04-13.54)$ & \\
\hline Residual disease & & & 0.22 & & & 0.31 \\
\hline None detectable & 1.00 (Ref) & $1.74(1.05-2.88)$ & & 1.00 (Ref) & $1.68(0.97-2.91)$ & \\
\hline$\leq 1 \mathrm{~cm}$ & 1.00 (Ref) & $0.94(0.45-1.94)$ & & 1.00 (Ref) & $0.96(0.47-1.99)$ & \\
\hline$>1 \mathrm{~cm}$ & 1.00 (Ref) & $2.17(1.04-4.51)$ & & 1.00 (Ref) & $1.87(0.91-3.84)$ & \\
\hline Comorbidity & & & 0.10 & & & 0.32 \\
\hline Yes & 1.00 (Ref) & $1.57(0.94-2.60)$ & & 1.00 (Ref) & $1.30(0.77-2.21)$ & \\
\hline No & 1.00 (Ref) & $1.44(0.88-2.35)$ & & 1.00 (Ref) & $1.53(0.92-2.53)$ & \\
\hline
\end{tabular}


Table 4. Hazard ratio $(95 \% \mathrm{Cl})$ for progression-free survival and overall survival among epithelial ovarian cancer patients according to the most recent preoperative serum sodium levels measured in the 90 days prior to surgery

\begin{tabular}{|c|c|c|c|c|c|c|}
\hline & \multicolumn{3}{|c|}{ Progression-free survival } & \multicolumn{3}{|c|}{ Overall survival } \\
\hline & Normal & Hyponatremia & $P$ value for & Normal & Hyponatremia & $P$ value for \\
\hline & HR $(95 \% \mathrm{CI}) \dagger$ & HR $(95 \% \mathrm{CI}) \dagger$ & Interaction & HR $(95 \% \mathrm{CI}) \dagger$ & HR $(95 \% \mathrm{CI}) \dagger$ & Interaction \\
\hline All patients & 1.00 (Ref) & $1.58(1.12-2.23)$ & & 1.00 (Ref) & $1.45(1.02-2.07)$ & \\
\hline FIGO stage & & & 0.06 & & & 0.05 \\
\hline I & 1.00 (Ref) & $1.24(0.25-6.09)$ & & 1.00 (Ref) & $2.30(0.46-11.38)$ & \\
\hline II & 1.00 (Ref) & $1.94(0.84-4.50)$ & & 1.00 (Ref) & $2.42(1.00-5.84)$ & \\
\hline III & 1.00 (Ref) & $1.19(0.76-1.86)$ & & 1.00 (Ref) & $0.97(0.60-1.55)$ & \\
\hline IV & 1.00 (Ref) & $3.54(0.90-13.96)$ & & 1.00 (Ref) & $3.03(0.87-10.52)$ & \\
\hline Residual disease & & & 0.23 & & & 0.38 \\
\hline None detectable & 1.00 (Ref) & $1.90(1.17-3.08)$ & & 1.00 (Ref) & $1.65(0.97-2.82)$ & \\
\hline$\leq 1 \mathrm{~cm}$ & 1.00 (Ref) & $0.94(0.46-1.95)$ & & 1.00 (Ref) & $0.96(0.46-1.97)$ & \\
\hline$>1 \mathrm{~cm}$ & 1.00 (Ref) & $2.14(1.03-4.44)$ & & 1.00 (Ref) & $1.65(0.81-3.38)$ & \\
\hline Comorbidity & & & 0.07 & & & 0.32 \\
\hline Yes & 1.00 (Ref) & $1.77(1.09-2.89)$ & & 1.00 (Ref) & $1.33(0.80-2.22)$ & \\
\hline No & 1.00 (Ref) & $1.43(0.87-2.34)$ & & 1.00 (Ref) & $1.52(0.92-2.52)$ & \\
\hline
\end{tabular}

CI, confidence interval; FIGO, International Federation of Gynecology and Obstetrics; HR, hazard ratio.

tHRs (95\% CIs) for progression-free survival and overall survival were estimated by using multivariable proportional hazard models that were adjusted for age at diagnosis, FIGO, residual disease, performance status, ascites, grading, and preoperative serum potassium level.

The severity of hyponatremia was found to be an independent factor predicting significantly higher in-hospital mortality [16]. Subsequently, Martin and colleagues [11] performed a retrospective population-based cohort study of women with a postoperative diagnosis of ovarian, fallopian tube, or primary peritoneal cancer who had undergone a cytoreductive procedure in the National Surgical Quality Improvement Program database from 2005 to 2013. Unfavorable prognosis of postoperative 30-day mortality and morbidity was observed among patients with hyponatremia compared with patients with normal sodium levels [11]. Although we focused on the relatively long-term outcome, the direction of the association was consistent with these published studies.

Previous studies estimated that about $14 \%$ of cases of hyponatremia among medical inpatients resulted from underlying tumor-related conditions and the prevalence differed depending on the race, region, and cancer type [17]. A study conducted on lymphoma, breast cancer, colorectal cancer, and lung cancer patients observed high prevalence rates of hyponatremia of $60 \%, 37 \%, 64 \%$, and $76 \%$, respectively [10]. Furthermore, an earlier study on 3,357 cancer patients admitted to the M.D. Anderson Cancer Center during a 3-month period in 2006 identified $47 \%$ of these patients as hyponatremic [7]. However, notably, the prevalence of EOC in these two studies was completely different. The prevalence of hyponatremia was $58.3 \%(28 / 48)$ in a study by Abu and coworkers [16], compared with $10.02 \%$ in a study by Martin and colleagues [11]. This difference might be partly attributed to the different cut-off values used in the studies. The cut-off value for hyponatremia used in the study by Abu and coworkers [16] was 130 $\mathrm{mmol} / \mathrm{L}$, while this value was $134 \mathrm{mEq} / \mathrm{L}$ for the study by Martin and colleagues [11]. It should also be considered that because cancer patients are frequently hyponatremic especially in the advanced stages, EOC has an asymptomatic course and the majority of patients have advanced-stage disease at the time of presentation. Consistent with this, the rates of prevalence of hyponatremia associated with FIGO stages I, II, III, and IV were $10.6 \%, 15.2 \%, 13.7 \%$, and $17.1 \%$, respectively, in this study.

Recently, several biological factors have been highlighted that may play a role in the development of hyponatremia in cancer patients. Low appetite, cachexia, and weight loss may induce malnutrition, with reduced sodium levels resulting from decreased oral intake. Furthermore, the high tumor burden, remote metastasis, and leaching of ascites into the peritoneal cavity of advanced malignancies may disturb the sodium balance [18] and induce vomiting, diarrhea, and renal fluid and solute losses in cancer patients, giving rise to hypovolemic hyponatremia [19]. In addition to malignancies, hyponatremia is seen in various medical conditions such as heart, kidney, and liver failure, as well as following the use of some medications, and the existence of one or more comorbidities increases the risk of hyponatremia [20]. Nevertheless, we observed no significant difference between preoperative serum sodium levels and the presence of comorbidities in the present study (Table 1). Furthermore, it was reported that hyponatremia in cancer patients is usually caused by SIADH, which may be driven by ectopic production of arginine vasopressin (AVP) by tumor tissues or by the effects of anticancer and palliative medications on AVP production or action [21]. It was reported that the tumors that were most frequently accompanied by hyponatremia of the SIADH types were lung, breast, and head and neck tumors [22].

The present study has several strengths. It was the first, as well as the largest, study exploring the 
prognostic value of hyponatremia in EOC in Asia, as well as in China. Furthermore, we carried out numerous subgroup analyses stratified by well-established prognostic factors as well as sensitivity analyses to assess whether the main findings were robust. Specifically, we considered not only the preoperative serum sodium levels as average values but also considered the most recent value measured in the 90 days prior to surgery, to check whether a possible correction had occurred and the potential influence of normalization.

This study also had several limitations that should be acknowledged. The first was its retrospective nature, meaning that potential recall and confounding bias might exist. However, in 2014, the Shengjing Hospital of China Medical University became the only "Dual Seven Level" hospital in China reaching level 7 of the Electronic Medical Record System Application Capacity Grading by the Hospital Management Institute of the National Health and Family Planning Commission and level 7 (the highest level) of the Electronic Medical Record Application Evaluation by the American Organization Healthcare Information and Management Systems Society. These achievements indicated the information systems in place in our hospital met the highest national and international standards, thereby minimizing the likelihood of recall bias. Furthermore, we addressed potential confounding bias by adjusting for all relevant covariates through regression models. Second, relatively short follow-up periods (median=3.1 years) and the high number of patients with missing information for variables or covariates $(n=288)$ was a concern in this study. However, although no differences were found between the included and excluded patients in this study, patients excluded due to their lack of serum sodium measurements were more likely to have normal serum sodium levels. Therefore, by including these patients, the results might have been biased towards the null hypothesis. Third, it should be noted that FIGO stage I accounted for a higher proportion of cases $(26.5 \%)$ compared with other studies. This phenomenon may be attributed to the accidental diagnosis of EOC when checking for other diseases such as benign ovarian tumors as well as myoma. Furthermore, the Department of Obstetrics and Gynecology of China Medical University is the highest authority on EOC diagnosis in northeast China, potentially explaining the early diagnosis of EOC in the present study.

In conclusion, our findings indicated that preoperative hyponatremia was an independent prognostic factor for EOC. Prospective studies are now warranted to confirm these findings. Our study highlighted the importance of early correction of hyponatremia in EOC patients because significantly improved survival was observed in the corrected group compared with other cancer patients [23,24]. Therefore, further interventional studies are also needed to investigate this issue in more depth.

\section{Acknowledgements}

\section{Funding}

This study was supported by grants from the Natural Science Foundation of China (No. 81602918 to Qi-Jun $\mathrm{Wu}$ ), the Doctoral Start-up Foundation of Liaoning Province (No. 201501007 to Qi-Jun Wu), the Younger research fund of Shengjing Hospital (Grant 2014sj09 to Qi-Jun Wu), and the Outstanding Youth Foundation of China Medical University (No. YQ20170002 to Qi-Jun Wu).

Qi-Jun $\mathrm{Wu}$ was supported by the Fogarty International Clinical Research Scholars and Fellows Support Center at the Vanderbilt Institute for Global Health, funded by the Fogarty International Center, NIH, through an R24 Training Grant (D43 TW008313 to Xiao-Ou Shu).

\section{Author Contributions}

J-YG and T-TG contributed equally to this work. Study concepts: Q-JW. Study design: J-YG, T-TG, YL, LW, Y-NW, and Q-JW. Data acquisition: J-YG, YL, LW, and Y-NW. Quality control of data and algorithms: T-TG and Q-JW. Data analysis and interpretation: J-YG, T-TG, ZY, and Q-JW. Statistical analysis: T-TG and Q-JW. Manuscript preparation: J-YG and Q-JW. Manuscript editing: J-YG and Q-JW. Manuscript review: J-YG, T-TG, ZY, YL, LW, Y-NW, and Q-JW.

\section{Competing Interests}

The authors have declared that no competing interest exists.

\section{References}

[1] Jayson GC, Kohn EC, Kitchener HC, et al. Ovarian cancer. Lancet 2014;384:1376-88.

[2] Zwakman N, van de Laar R, Van Gorp T, et al. Perioperative changes in serum ca125 levels: a prognostic factor for disease-specific survival in patients with ovarian cancer. Journal of Gynecologic Oncology 2017;28:e7.

[3] Ayhan A, Gunakan E, Alyazici I, et al. The preoperative albumin level is an independent prognostic factor for optimally debulked epithelial ovarian cancer. Archives of Gynecology and Obstetrics 2017;296:989-95.

[4] Ge LN, Wang F. Prognostic significance of preoperative serum albumin in epithelial ovarian cancer patients: a systematic review and dose-response meta-analysis of observational studies. Cancer Management and Research 2018;10:815-25.

[5] Li J, Jiao X, Yuan Z, et al. C-reactive protein and risk of ovarian cancer: a systematic review and meta-analysis. Medicine (Baltimore) 2017;96:e7822.

[6] Whelan B, Bennett K, O'Riordan D, et al. Serum sodium as a risk factor for in-hospital mortality in acute unselected general medical patients. QJM 2009;102:175-82.

[7] Doshi SM, Shah P, Lei X, et al. Hyponatremia in hospitalized cancer patients and its impact on clinical outcomes. American Journal of Kidney Diseases 2012;59:222-28. 
[8] Mohan S, Gu S, Parikh A, et al. Prevalence of hyponatremia and association with mortality: results from nhanes. American Journal of Medicine 2013;126:1127-37.

[9] Balachandran K, Okines A, Gunapala R, et al. Resolution of severe hyponatraemia is associated with improved survival in patients with cancer. Bmc Cancer 2015;15:163.

[10] Castillo JJ, Glezerman IG, Boklage SH, et al. The occurrence of hyponatremia and its importance as a prognostic factor in a cross-section of cancer patients. Bmc Cancer 2016;16:564.

[11] Martin JY, Goff BA, Urban RR. Preoperative hyponatremia in women with ovarian cancer: an additional cause for concern? Gynecologic Oncology 2016;142:471-76.

[12] Benedet JL, Bender H, Jones HR, et al. Figo staging classifications and clinical practice guidelines in the management of gynecologic cancers. Figo committee on gynecologic oncology. Int J Gynaecol Obstet 2000;70:209-62.

[13] Duska LR, Kohn EC. The new classifications of ovarian, fallopian tube, and primary peritoneal cancer and their clinical implications. Annals of Oncology 2017;28:i8-12.

[14] Oken MM, Creech RH, Tormey DC, et al. Toxicity and response criteria of the eastern cooperative oncology group. Am J Clin Oncol 1982;5:649-55.

[15] Charlson ME, Pompei P, Ales KL, et al. A new method of classifying prognostic comorbidity in longitudinal studies: development and validation. J Chronic Dis 1987;40:373-83.

[16] Abu ZG, Al-Kindi SG, Hassan AA, et al. Hyponatraemia in cancer: association with type of cancer and mortality. Eur J Cancer Care (Engl) 2015;24:224-31.

[17] Gill G, Huda B, Boyd A, et al. Characteristics and mortality of severe hyponatraemia--a hospital-based study. Clin Endocrinol (Oxf) 2006;65:246-49.

[18] Onitilo AA, Kio E, Doi SA. Tumor-related hyponatremia. Clin Med Res 2007;5:228-37.

[19] Fiordoliva I, Meletani T, Baleani MG, et al. Managing hyponatremia in lung cancer: latest evidence and clinical implications. Therapeutic Advances in Medical Oncology 2017;9:711-19.

[20] Adrogue HJ, Madias NE. Hyponatremia. N Engl J Med 2000;342:1581-89.

[21] Castillo JJ, Vincent M, Justice E. Diagnosis and management of hyponatremia in cancer patients. Oncologist 2012;17:756-65.

[22] Berghmans T, Paesmans M, Body JJ. A prospective study on hyponatraemia in medical cancer patients: epidemiology, aetiology and differential diagnosis. Supportive Care in Cancer 2000;8:192-97.

[23] Berardi R, Santoni M, Newsom-Davis T, et al. Hyponatremia normalization as an independent prognostic factor in patients with advanced non-small cell lung cancer treated with first-line therapy. Oncotarget 2017;8:23871-79.

[24] Balachandran K, Okines A, Gunapala R, et al. Resolution of severe hyponatraemia is associated with improved survival in patients with cancer. Bmc Cancer 2015;15:163. 